\title{
Verantwortung gegenüber der Gesellschaft als Unternehmensstrategie
}

\section{Zum Stand von »Corporate Social Responsibility « in Großbritannien}

Felix Behling

Felix Behling studiert Soziologie an der Universität Essex in Großbritannien.

E-Mail fbehli@essex.ac.uk
Unternehmerische Verantwortlichkeit ist ein anerkannter Bestandteil im $\mathrm{Zu}$ sammenspiel von Politik, Gesellschaft und Wirtschaft in Großbritannien. Beispielsweise sind alle an der Börse vertretenen Unternehmen angehalten, regelmäßig über ihr gesellschaftliches Engagement zu berichten.

»Every little helps' can become a great deal when everyone pulls in the same direction."

Sir Terry Leahy, Chief Executive Tesco Plc

Wenn von unternehmerischer Verantwortung in Großbritannien gesprochen wird, dann geschieht dies häufig unter der Überschrift von »Corporate Social Responsibility «(CSR). Es handelt sich dabei um all jene unternehmerischen Aktivitäten, die in Bezug zu verschiedenen Interessengruppen unternommen werden und in Verbindung zum gewinnorientierten Handeln stehen.

»Every little helps «, die Werbebotschaft und zugleich das Motto der CSRAktivitäten von Tesco, des größten britischen Einzelhandelsunternehmens, verdeutlicht, dass die Verantwortung zum beiderseitigen Nutzen von Gesellschaft und Unternehmen beitragen soll. Die unternehmerische Verantwortung in Großbritannien ist tiefer und in einem liberaleren Wirtschaftsverständnis verankert. Beides mündet in einer andersartigen, aber gleichzeitig doch ähnlichen Ausprägung als in Deutschland. Die These zum Status der gesellschaftlichen Verantwortung britischer Unternehmen lautet daher: Unternehmerische Verantwortlichkeit ist ein anerkannter Bestandteil im Zusammenspiel von Politik, Gesellschaft und Wirtschaft.

Die akademische Debatte um unternehmerische Verantwortung in Großbritannien orientiert sich zu einem großen Teil an der US-amerikanischen Diskussion. Dies kann zum einen durch die sprachliche Nähe und zum anderen durch die ähnlichen Wirtschaftssysteme erklärt werden. Die inhaltliche Bedeu- tung von unternehmerischer Verantwortung hat sich dabei im Laufe der Jahre jedoch grundsätzlich verändert. Wurde Verantwortlichkeit bis in die 1960er noch im Sinne von Philanthropie und Patriarchat diskutiert, wird soziale Verantwortung gegenüber der Gesellschaft nun als Teil der Unternehmensstrategiestrategie verstanden, die in Verantwortung für eine effizientere und nachhaltigere Wertschöpfungskette steht.

Auf der institutionellen Ebene hat sich dieser Wechsel in der Formierung der Nichtregierungsorganisation "Business in the Community « vollzogen, die seit 1982 eine Plattform zum Austausch über und zur Koordination von Verantwortung zwischen einzelnen Unternehmen und zudem Unterstützung im Aufbau von Programmen sowie deren Einbindung in die Geschäftsstrategie anbietet. »Business in the Community « ist nun auch für die Umsetzung des reformierten Unternehmensgesetzes aus dem Jahre 2006 zuständig. Die britische Regierung, vor allem das Wirtschaftsministerium, verfolgt seit zehn Jahren eine Politik der Nachhaltigkeit. Das Gesetz stellt einen vorläufigen Höhepunkt dar, da alle an der Börse vertretenen Unternehmen dazu verpflichtet sind, über Aspekte gesellschaftlichen Engagements zu berichten. Vor diesem Hintergrund kann sicherlich argumentiert werden, dass unternehmerische Verantwortung institutionell, politisch sowie akademisch sichtbar verankert ist und Großbritannien eine Vorreiterrolle in Europa hat.

Wie wird unternehmerische Verantwortung definiert? Welche Maßnahmen werden ergriffen, um dieser gerecht zu werden? Wie ist Verantwortlichkeit wirtschaftlich verankert? Und vor allem, wie wird das Engagement in der Gesellschaft wahrgenommen?

Die historische Besonderheit des britischen Wirtschaftssystems erschwert eine endgültige Beantwortung dieser Fragen: 
- Zum Ersten wurden große Teile der Wirtschaft, die sich vormals im staatlichen Besitz oder unter dessen Kontrolle befanden, seit der Regierung Thatcher entweder durch Privatisierung oder Private Public Partnerships dereguliert. Dies betrifft zum Beispiel nicht nur kommunale Versorger oder die Gesundheitsversorgung, sondern auch Institutionen industrieller Beziehungen wie Gewerkschaften.

- Infolgedessen rückte zum Zweiten der Umgang mit Unternehmen auf der ideologischen Ebene wieder näher zu einer liberaleren Auffassung. Das aktuelle Unternehmensgesetz verlangt zwar einen jährlichen Bericht über Aktivitäten, aber es schreibt diese nicht vor. Diese Berichtpflicht führt in Einzelfällen jedoch dazu, dass rechtlich vorgeschriebene Maßnahmen als unternehmensspezifische Aktivitäten des Corporate Social Responsibility gekennzeichnet werden. Alle Unternehmen beschreiben sich zum Beispiel als Arbeitgeber mit einer moralischen Selbstverpflichtung zur Gleichbehandlung aller Mitarbeiterinnen und Mitarbeiter; ein entsprechendes Antidiskriminierungsgesetz wurde bereits 1995 verabschiedet und 2005 wesentlich erweitert. Das britische Rechtssystem basiert zudem auf Präzedenzfällen, so dass dieses im Grunde eher einen empfehlenden statt regulierenden Charakter hat. Direkte legislative und politische Eingriffe, wie zum Beispiel das Antidiskriminierungsgesetz, werden entweder vermieden oder nur vorsichtig vorgenommen, da diese wirtschaftlich, politisch und gesellschaftlich ambivalent aufgenommen werden.

- Drittens konzentrieren sich mehr als dreißig Prozent des britischen Wirtschaftswertes auf London sowie Südostengland, ergänzt durch den Großraum Manchester und die Region Edinburgh-Glasgow. Wirtschaftliches Handeln in England ist somit zu einem Teil immer direkt oder indirekt von Entwicklungen in London abhängig oder wird von diesen geprägt. Zentren mit einer ähnlichen wirtschaftlichen Bedeutung gibt es in Deutschland sicherlich nur im Rhein-Ruhr-Gebiet und im Großraum Frankfurt am Main.

Obwohl diese drei Faktoren eine endgültige Antwort auf die genannten Fragen erschweren, lässt sich der Stand unternehmerischer Verantwortung in Großbritannien ansatzweise skizzieren.

Britische Unternehmen sehen sich verantwortlich, wenn ihre wirtschaftlichen Aktivitäten Interessengruppen betreffen. Mit den verschiedenen CSR-Programmen versuchen sie vorausschauend zum einen die zukünftige Wettbewerbsfähigkeit und zum anderen ihre Selbstregulierung zu gewährleisten. Die Unternehmen definieren gesellschaftliche Verantwortung aber nicht als Altruismus, sondern betten sie häufig in die Maximierung des Shareholder Value ein. Unternehmen beziehen sich auf vier Verantwortungsbereiche, deren Bedeutung jeweils variiert; diese sind: Umwelt, Mitarbeiter, Gesellschaft und Zulieferer.
Schulen lösen dann wiederum die Gutscheine bei Tesco für technische Geräte ein. Tesco vergütet jeden Gutschein mit einem Wert von 0,001 Pence. Obwohl es gering erscheint, werden pro Jahr Geräte im Wert von 6,5 Millionen Pfund an Schulen ausgegeben und Tesco nimmt theoretisch nur eine vermittelnde Position ein, da am Ende der Kunde die Gutscheine verteilt.

Unternehmerische Verantwortung scheint wirtschaftlich tief verankert $\mathrm{zu}$ sein, weil die Mehrzahl der börsennotierten Unternehmen jährliche Berichte über ihre CSR-Aktivitäten veröffentlicht und sie die Reporte auch durch Beratungsfirmen zertifizieren lassen. Die Begutachtung ist teilweise standardisiert und orientiert sich an international erarbeiteten

\section{"Britische Unternehmen kennen neben dem Shareholder Value vier Verantwortungsbereiche: Umwelt, Mitarbeiter, Gesellschaft, Zulieferer «}

Die Bereiche spiegeln zunächst die deutsche Situation wider, die gleiche Schwerpunkte hat. Britische wie auch deutsche Unternehmen nehmen ihre Verantwortung entweder in monetärer oder nicht-monetärer Form wahr. Zum einen stellen sie finanzielle Mittel zur Verfügung, um zum Beispiel die ökologische und soziale Regenerierung von Bergbaugebieten zu gewährleisten, wie es von Anglo-American, einem Montanunternehmen, in Nordengland durchgeführt wird. Zum anderen stellen sie aber auch Ressourcen wie Fachkräfte oder betriebliche Strukturen bereit, um wohlfahrtliche Programme zu unterstützen. Ähnlich der Initiative »Schulen ans Netz« der Deutschen Telekom bietet Tesco Schulen die Möglichkeit, Computer $\mathrm{zu}$ einem niedrigen Preis zu beziehen. British Telecom und das Innenministerium unterstützen Tesco in dieser Aufgabe. Anders als in Deutschland ist dieses Programm aber vollkommen auf lokaler Ebene und durch Kunden organisiert. Kunden bekommen je nach Höhe des Einkaufes Gutscheine, die sie an Schulen ihrer Wahl weitergeben können. Diese freiwilligen Verfahren, wie zum Beispiel der Global Reporting Initiative (GRI) der Vereinten Nationen oder dem Account Ability Assurance Standard. Dazu gehört unter anderem die Aufstellung von Zielen, deren Erfolg sich anhand von Indikatoren leicht überprüfen lässt. Alle 100 börsennotierten größten Unternehmen haben zudem eigenständige CSR-Abteilungen, die unternehmerische Verantwortung koordinieren und in den technischen sowie kaufmännischen Unternehmensbereichen umsetzen. In Verbindung mit den bereits genannten Beispielen bestätigt dies die Annahme, dass unternehmerische Verantwortung in Großbritannien bereits stärker in die strategische Planung einbezogen wird.

Im Gegensatz zu deutschen Unternehmen haben in britischen Firmen die Mitarbeiter - und somit die innerbetriebliche Verantwortung - einen weitaus höheren Stellenwert. Die unternehmerische Verantwortung bezieht sich ebenfalls auf Mitarbeiter, weil sie eine andere Interessengruppe darstellen; es wird ferner deutlich, dass Corporate Social Responsibility entgegen der vielfachen Wahrnehmung 
nicht nur externe sondern auch interne Interessengruppen eines Unternehmens betrifft.

Für die größere Bedeutung innerbetrieblicher Verantwortung in Großbritannien sollen hier zwei Gründe genannt werden:

- Erstens ist der Einfluss von Gewerkschaften seit der Regierung Thatcher gesunken und die Mehrheit der Mitarbeiter ist nicht mehr gewerkschaftlich vertreten oder durch ähnliche Gesetze geschützt.

- Zum Zweiten basiert die Altersvorsorge mehrheitlich auf privaten Initiativen, obgleich eine niedrige gesetzliche Grund- rente garantiert ist. Britische Unternehmen bieten ihren Mitarbeitern entweder durch eigene oder fremde Pensionsfonds private Altersvorsorge an. Neben Investmentfonds sind Pensionsfonds die größten Anteilseigner der im Aktienindex gelisteten Unternehmen.

Die Verantwortung in Bezug auf Mitarbeiter konzentriert sich dabei neben der finanziellen Teilhabe am Unternehmen auf eine größere Partizipation in Entscheidungsprozessen, Gleichbehandlung aller Mitarbeiter, Weiterbildung und Arbeitsschutz. Es gibt dennoch Querverbindungen zwischen inner- und außerbetrieblicher Verantwortung. Obwohl die

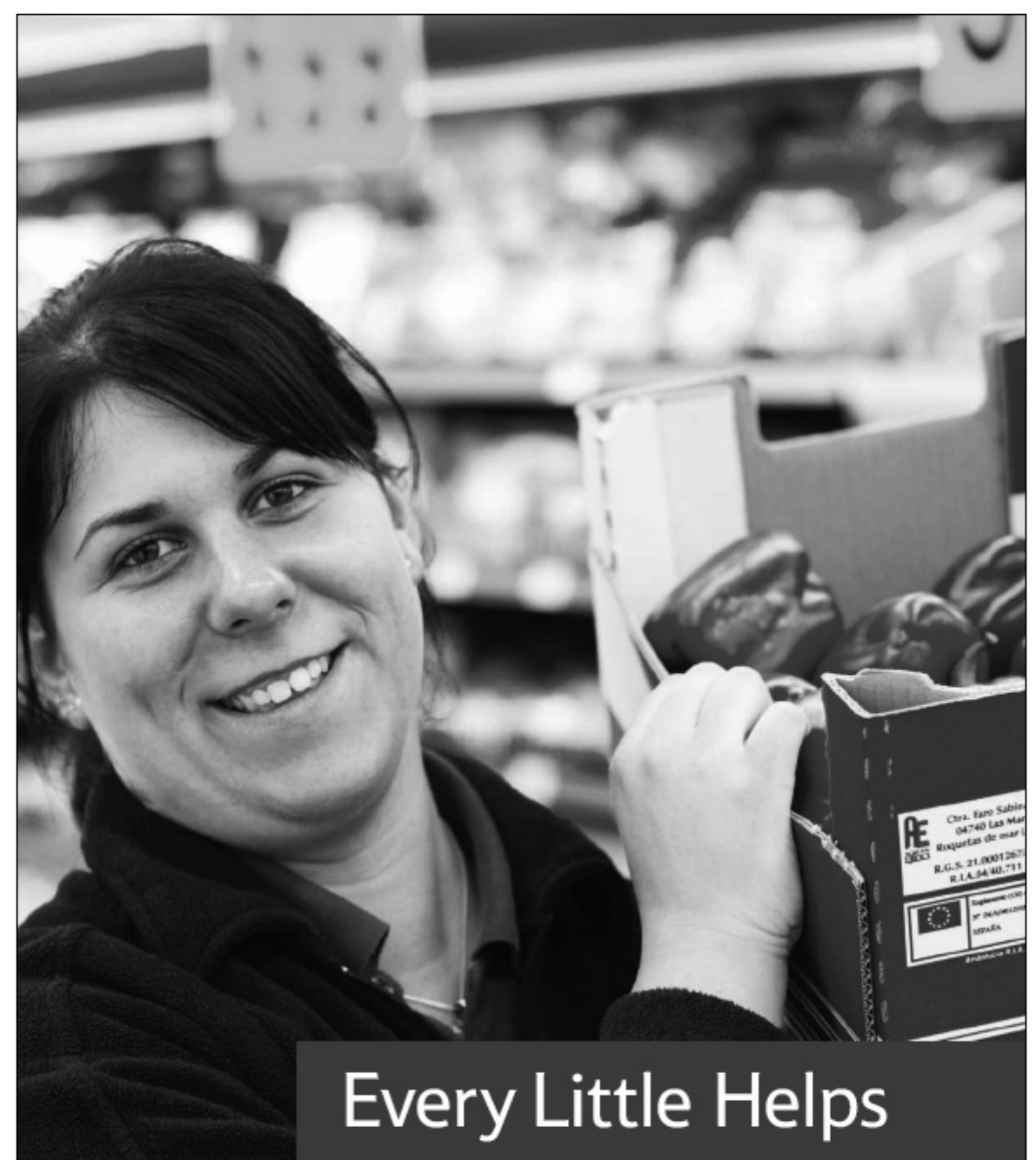

www.tesco.com/crreview08 seit Januar 2008 zulässige Vergabe einer staatlich anerkannten Hochschulreife durch McDonalds Großbritannien im Zuge einer Ausbildung im Restaurant durchaus Fragen aufwirft, muss festgehalten werden, dass damit auch Möglichkeiten zu einer weiteren Bildung für sonst vielleicht benachteiligte Bevölkerungsschichten geschaffen werden und $\mathrm{McDo}$ nald's die Effizienz der Mitarbeiter erhöhen kann.

\section{Resümee}

Unternehmerische Verantwortung in Großbritannien ist ähnlich und doch verschieden zu der in Deutschland. Durch die sprachliche Nähe zu den USA konnte sich die Debatte über verantwortliches Handeln bereits früh entfalten und institutionalisieren. Das hat dazu geführt, dass Unternehmen ihre Rolle eher akzeptieren und in die Geschäftsstrategie einbinden konnten. Vier Bereiche - Umwelt, Mitarbeiter, Gesellschaft und Zulieferer werden dabei ähnlich wie in Deutschland abgedeckt. Im Unterschied haben aber die Mitarbeiter einen höheren Stellenwert, da gesetzliche Regelungen in einem geringeren Umfang als in Deutschland vorhanden sind und die Rolle der Gewerkschaften unklarer ist.

Mit einer Beobachtung der britischen Presse kann gesagt werden, dass Unternehmen und ihr Handeln akzeptierter sind. Zwar wird bestimmtes Verhalten wie Tescos Pläne zur Steuerersparnis ebenfalls kritisch aufgenommen, aber im weiteren Kontext wird Unternehmen das Recht auf freies Handeln zuerkannt. Dies spiegelt das Wirtschaftssystem im Generellen wider.

Eine endgültige und eindeutige Beurteilung des Standes unternehmerischer Verantwortung in Großbritannien erscheint fast unmöglich, da verschiedene Faktoren in einer besonderen Konstellation zusammenwirken. Es lässt sich festhalten, dass die gesellschaftliche Verantwortung in Großbritannien etwas fortgeschrittener ist. Wie in Deutschland nehmen Unternehmen ihre Verantwortlichkeit in verschiedenen Bereichen und Ebenen wahr. Die Verantwortung ist jedoch bereits stärker in die Geschäftsstrategie eingebunden und einzelne Komponenten wie Mitarbeiter haben eine größere Bedeutung. 